\title{
Comprehensive Glaucoma Management: Importance of Follow-up
}

\author{
${ }^{1}$ Rajul S Parikh, ${ }^{2}$ Shefali R Parikh \\ ${ }^{1}$ Bombay City Eye Institute and Research Center, Mumbai, Maharashtra, India \\ ${ }^{2}$ Lotus Eye Hospital, Mumbai, Maharashtra, India
}

The glaucomas are not a single disease entity but result from different mechanisms that eventually lead to loss of retinal ganglion cells. The terminal event in the glaucoma continuum is irreversible visual impairment and blindness. All the resources for the management of glaucoma are directed towards arresting further RNFL (retinal nerve fiber layer) loss so as to prevent further visual deterioration and preserve the quality of life for the rest of the patient's life span. It is a slowly progressive chronic disease and regular follow-up is necessary to monitor the disease stability. It is also important to have regular followup to detect progression in earlier stage that might require escalation of treatment.

The objectives of assessment during follow-up visits are:

1. To determine whether glaucoma is stable or progressing

2. If disease is progressing then to identify underlying mechanism for progression and change treatment (management plan) accordingly.

Following are the factors we must evaluate during followup visit. However we must remember that every individual is unique and management plan needs to be individualized.

Progression in glaucoma is assessed by structure (optic disk and RNFL) and function (visual field testing with white on white perimetry) independently or in association. A higher IOP is a risk factor for progression. Structure, function and IOP should be monitored at regular intervals. The follow-up period depends on the stage of the disease and stability.

\section{SPECIFIC POINTS DURING FOLLOW-UP VISITS}

\section{Re-evaluation of IOP and Target IOP during Follow-up}

The target IOP requires periodic re-evaluation. During the re-evaluation, we need to decide whether the target is appropriate and should not be changed, or the target needs to lowered, or that the target should be raised. It depends upon the presence or absence of glaucomatous progression, the effect of the therapy upon the patient's HRQOL (health related quality of life), and whether the patient has developed any new systemic illness that might affect the risk/benefit ratio of therapy or likely to greatly shorten life expectancy. The presence or absence of progression is determined by serial assessment of the optic nerve head and/or retinal nerve fiber layer and of the functional damage on perimetric testing.

\section{Gonioscopic Changes}

It should maintain baseline examination conditions.

We must perform gonioscopy regularly in patients with angle closure (6/12 to at least once a year) and periodically in patients with open angles (once in 2 years). This must be performed when you suspect change in clinical picture. It is very important to maintain baseline gonioscopic conditions. In angle closure glaucoma, increase in appositional and/or synechial closure is a sign of progressive disease and it must be looked carefully every time we perform gonioscopy.

\section{Individualizing Follow-up Visits}

If the unilateral drug trial has been started, we review patients depending on the peak duration of the drug used. Subsequent follow-up is customized depending on the decision to continue the same drug, add or replace the drug in the same eye and the other eye also.

At each exam, record dosage and frequency of use, discuss adherence to the therapeutic regimen and patient's response to recommendations for therapeutic alternatives or diagnostic procedures. We must ask about any new systemic disease or change in current systemic medications during each follow-up visits.

Once we are sure that IOP is stable (assessed by day DVT or checking IOP at frequent intervals at different hours of the day at different visits), the subsequent follow-up visits of glaucoma patients depends on the severity of glaucoma (amount of disk and field damage) at the time of presentation and followup, as well as additional risk factors. The initial visits are used to obtain baseline fields. 
Glaucoma (preperimetric or early functional damage), with IOP at target might be reviewed in 4-6 months, and then if still stable, at half yearly intervals. The yearly follow-ups would include the full comprehensive eye examination as well as several IOP's, visual fields and other imaging tests required.

Stable patients with moderate damage would be examined at 3 to 6 month intervals. For severe glaucoma in the better eye, the interval could be 3-4 months. If the IOP is slightly higher than the target level, say $2 \mathrm{~mm} \mathrm{Hg}$ but the patient has not progressed on other parameters, we would schedule the next visit a few months earlier to measure IOP's and then go back to the routine. If there is confirmed progression in structure or function (repeatable progression of field defect on at-least two occasions), even if the office hour IOP is at target level, we repeat DVT to look for undetected IOP peaks or high fluctuations as well as systemic factors like nocturnal dips in blood pressure. While it is controversial, we believe that diurnal fluctuation of 8 $\mathrm{mm} \mathrm{Hg}$ or more is probably an independent risk factor for progression.

\section{Judging Progression}

Progression can be judged by documenting structural changes in the optic disk and or functional changes on the visual fields. IOP and optic disk examinations are mandatory at every routine visit.

How frequently assessment on functional and structural test should be done depending upon patient's initial damage. The current EGS guideline suggests that ideally at least 5-6 visual field should be performed in first 2 year to establish rate of progression (EGS guidelines). ${ }^{1,2}$ Subsequently frequent field should be done only for those patients who show rapid progression otherwise once a year perimetry would be suffice. We prefer 6 monthly optic disk imaging in early and moderate glaucoma.

\section{Progression in Structure}

Any new RNFL defect, presence of new disk hemorrhage or change in neuroretinal rim status indicates unstable and progressive glaucoma. This patient requires a visual field to document functional progression. Where baseline optic disk photographs and serial photography are available, detection of these changes is substantially enhanced. If photographs are not available, the pupil should be dilated for stereoscopic optic disk evaluation.

\section{Progression on Visual Fields}

In current clinical practice, we rely mainly on WWP to detect progression. In our own clinical routine: we use the "Overview" and glaucoma progression analysis (GPA) programs. ${ }^{3}$ Eyeballing the overview we get a feel for whether field deterioration is due to generalized sinking of the hill of vision or whether it is due to glaucoma. GPA is based on the pattern deviation plot and is better option to diagnose progression.

Progression in the research setting requires confirmation over 4-6 fields. ${ }^{4}$ In the clinic however, the judgment of progression is more "corroborative". There are other factors like the IOP (hopefully several, over time, at various times of the day), appearance of the disk, nerve fiber layer, hemorrhage, etc. If these suggest progression, eyeballing the overview gives us an idea as to what is going on and we get a GPA. "Possible" progression on the GPA corroborates our clinical concern; a repeat field with the message "Likely" progression is then probably good enough to take decisions, especially if the deteriorating points correlate with our disk findings. It is possible that GPA might underestimate visual field progression even in cases even without evidence of increasing media opacity. We feel that consideration of the overview program (total and pattern deviation plot) in combination with the GPA analyses addresses this concern. Newer VFI visual field index) may be beneficial as it also predicts the trend of worsening based on patient's past visual field record (Fig. 1).

\section{Progression on Imaging Technologies}

Imaging technique offer exciting possibilities to detect progression. ${ }^{5}$ The Heidelberg retina tomogram has the longest track record in documenting structural change (Trend analysis and topographic change analysis; Figure 2). Scanning Laser Polarimetry and OCT have developed programs more recently. Imaging technologies will be part and parcel of glaucomatologist in confirming progression of disease. Progression on imaging is more likely to be useful in the earlier stages of the disease, which is an advantage.

If the progression is confirmed on both structure and function or even if only on visual fields, there is no other option but to further reduce our target IOP by adding or substituting therapy.

Every thing else being equal, we take more cognizance of optic disk deterioration in the presence of a field defect than without. Some findings like disk hemorrhage, new nerve fiber layer defect, or a rim notch are so predictive of future field defects that we initiate our routine (DVT, detection of IOP dips, etc.) on this basis and would step up therapy without a field defect.

We treat progression on Imaging (Trend analysis and topographic change analysis on HRT) in the same manner. Without a field defect progression (unless associated with the disk findings referred to above, or "high" IOP), we would usually elect to observe the patient more carefully. In the presence of an existing functional defect (early, or moderate), the change is more likely to be real and result in functional damage. Accordingly we consider progression on imaging more sinister 


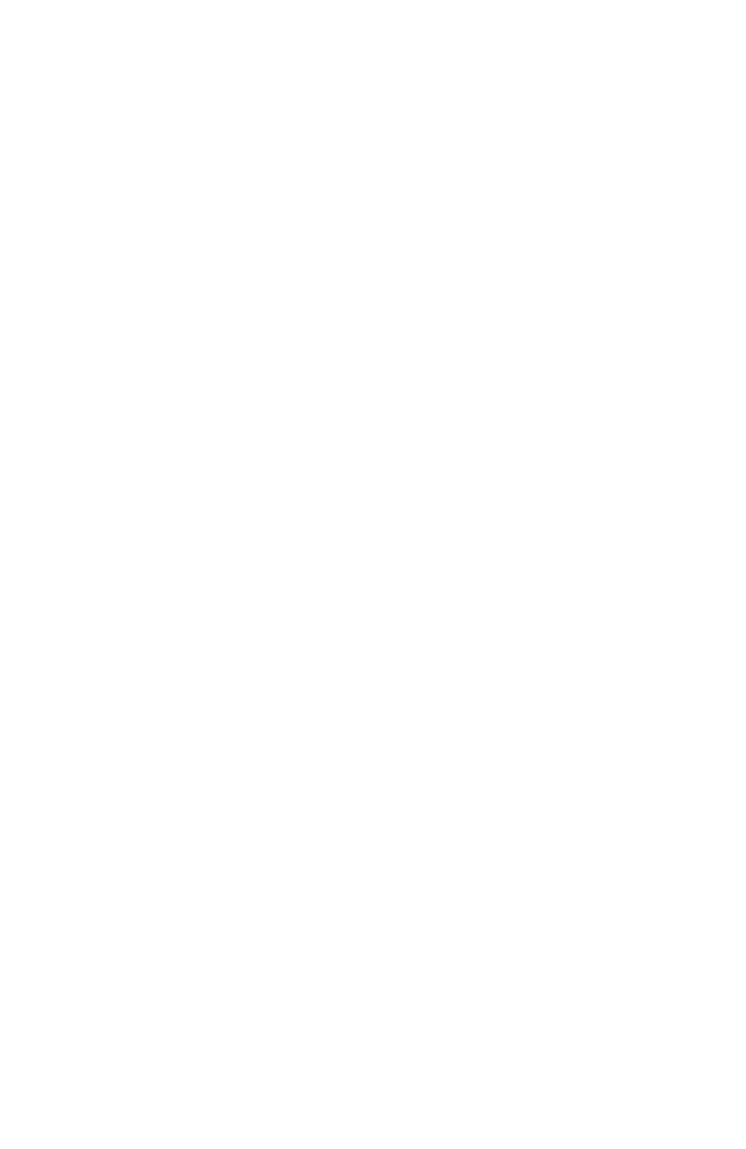

Fig. 1: VFI estimating rate of progression

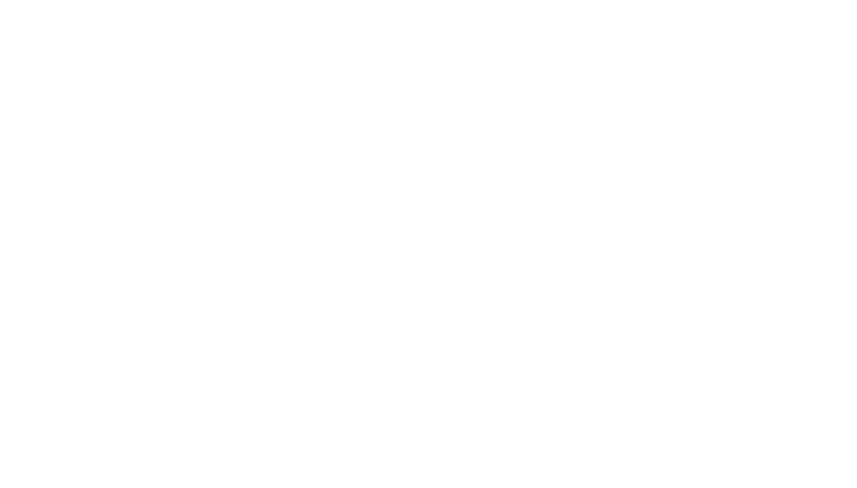

Fig. 2: HRT change probability map

in this situation and treat it akin to disk progression in this situation.

Either way, if target IOP was not achieved then in these cases we may add on or change the therapy

The patient's quality of life (QOL) should be estimated and the impact of the glaucoma management on QOL assessed.

This forms part of the assessment of burden of disease and burden of treatment. This is important as it has direct bearing with adherence to treatment and so to success of our management plan.

In essence, following issues need to be considered and evaluated during follow-up visits:

1. Discuss the patient's subjective well-being and visual function.

2. Reassess risk factors: IOP and gonioscopic change.

3. Reassess structure and function of the optic nerve.

4. Estimate rate of (any) progression.

5. Identify adverse effect(s) of treatment:

Adverse effects of treatment should be actively asked using general and specific questioning. These can include:

- General effects: Self-rated health, feelings about/attitude towards treatment.

- Systemic effects: Respiratory, cardiovascular, digestive, neurological.

- Local effects: Stinging/burning, blurring, itching, redness.

6. Assess adherence to treatment.

7. Identify change(s) in current medical and ophthalmic problems.

8. Discuss quality of life issue(s).

9. Reinforce appropriate patient information:

a. Revise management

b. Plan follow-up.

\section{Some Clinical Situations Merit Mention}

a. If the progression is confirmed but IOP is at target (confirmed with DVT): Revisit other risk factors like, nocturnal hypotension, systemic or topical steroid use, recent major surgery, central corneal thickness, etc. We would also talk to the patient's physician regarding any hypertension, it's control and the avoidance of night-time dips in blood pressure as well as the possibility of sleep apnea. If the patient shows progression with normal IOPs, we would enquire about other habits; yoga, especially "aasnas" in the inverted position, use of wind instruments, rapid consumption of large quantities of water or beer. An "alternative medicine" prescription of a liter of water in the morning mimics the water drinking Provocative test and can cause optic nerve damage. This situation also requires a repeat DVT to see if the IOP's are fluctuating beyond the normal range especially in the early morning hours. If 24 hour DVT also shows IOPs to be low teens than we may need to evaluate IOP independent factors, which we have mentioned in NTG section.

It is also possible that our assessment of the initial target IOP was wrong and we need to readjust it. On other hand, we may have a patient at the opposite end of the spectrum: mild or preperimetric glaucoma with stable visual functions at target. In this scenario, it is possible that we may have 
Table 1: Factors for nonadherence and strategies

\begin{tabular}{|c|c|}
\hline Barrier & Strategies to address \\
\hline $\begin{array}{l}\text { Lack of concern, } \\
\text { needs motivation }\end{array}$ & $\begin{array}{l}\text { Provide specific information } \\
\text { about glaucoma and its } \\
\text { consequences }\end{array}$ \\
\hline $\begin{array}{l}\text { - Problems taking medicines } \\
\text { several times a day }\end{array}$ & $\begin{array}{l}\text { Switch to medication with fewer } \\
\text { daily doses. Discuss strategies to } \\
\text { remember each dose }\end{array}$ \\
\hline - Concerned about side effects & $\begin{array}{l}\text { - Reassess current regimen if nature } \\
\text { of side effects warrant, shorten } \\
\text { time to patient's next visit }\end{array}$ \\
\hline $\begin{array}{l}\text { - Difficulty with drop } \\
\text { administration }\end{array}$ & $\begin{array}{l}\text { - Suggest delivery aids or support } \\
\text { group }\end{array}$ \\
\hline
\end{tabular}

overestimated the target IOP and we may be over treating the patient. In this instance we would try to withdraw unnecessary drugs.

b. Adherence: Glaucoma is unfortunately life long disease and if patient treated with medication would require like long medications. This brings the issue of adherence to medication, as quite few times it is nonadherence to medication, which is the reason for disease progression.

There can be various reasons for nonadherence and that can include patient factor and physician factor. ${ }^{6-8}$ For example, patient with Musculo-skeletal disorders like arthritis (osteorheumatoid) or CNS disease like Parkinsonism and other disease which affect hand coordination may affect severely the ability to administer eye drops and subsequently adherence to medical management.

Identification of nonadherence is difficult and there is no sure way we can identify.

We have adopted four step approach to identify nonadherence:

1. Begin with a direct open-ended question: "Tell me how you've been taking your medications"

2. Reverse the judgemental environment. Reassure that nonadherence is: Universal and normal or understandable

3. Enlist the patient's active participation: Make correct information as important as creating a good impression by explaining how the self-reported adherence information will be used

4. THEN, ask about "forgetting” or "missed" medications

We need to identify the root cause for nonadherence and then and then we can improve the adherence. Table 1 shows some factors and strategies.

\section{REFERENCES}

1. European Glaucoma Society Glaucoma guidelines; Terminology and Guidelines for Glaucoma; IIIrd Edition; 2009.

2. B C Chauhan BC, Garway-Heath DF,Goñi FJ, et al. Practical recommendations for measuring rates of visual field change in glaucoma. Br J Ophthalmol 2008;92:569-73.

3. Parikh R, Parikh S, Navin S, Ellen A, Thomas R. Practical aspect of medical Management of glaucoma: Review article. Indian Journal of Ophthalmology 2008;56:223-30.

4. M Schulzer and the Normal Tension Glaucoma study group. Errors in the Diagnosis of Visual Field Progression in normal - tension glaucoma. Ophthalmology 1994;101:1589-95.

5. Glaucoma Diagnosis: Structure and Function. Edited by Erik Grave, Robert Wienereb, 2004, Kurger publication, Hague, Netherlands.

6. Sabaté. Adherence to Long-term Therapies: Evidence for Action. Geneva, Switzerland: World Health Organization; 2003.

7. Maguire P, Pitceathly C. Key communication skills and how to acquire them. BMJ 2002;28:325(7366):697-700.

8. Haynes RB, Yao X, Degani A, et al. Interventions for enhancing medical adherence. Cochrane Database of Systemic Review, 2005.

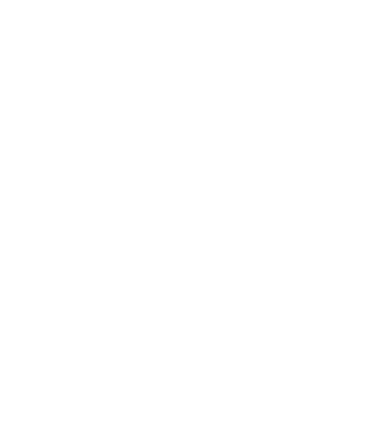

Rajul S Parikh

(drparikhs@gmail.com) 